\title{
Expression of toll-like receptors in hepatic cirrhosis and hepatocellular carcinoma
}

\author{
L. Sun ${ }^{1 *}$, J.J. Dai ${ }^{1 *}$, W.F. Hu ${ }^{1,2}$ and J. Wang ${ }^{1}$ \\ ${ }^{1}$ Department of Aetiology \& Immunology, Medical College, \\ Anhui University of Science \& Technology, Huainan, China \\ ${ }^{2}$ Clinical Laboratory of the Affiliated Cancer Hospital of Anhui \\ University of Science \& Technology, Huainan, China \\ *These authors contributed equally to this study. \\ Corresponding author: J. Wang \\ E-mail: wangjian8237@sina.com
}

Genet. Mol. Res. 15 (2): gmr.15027419

Received August 11, 2015

Accepted September 9, 2015

Published July 15, 2016

DOI http://dx.doi.org/10.4238/gmr.15027419

\begin{abstract}
Toll-like receptors (TLRs) can specifically identify pathogen-associated molecular patterns (PAMPs) by recognizing structural patterns in diverse microbial molecules, and can provide an effective defense against multiple microbial infectious. A variety of TLRs can be expressed on the surface of liver parenchymal as well as nonparenchymal cells. Kupffer cells are a type of hepatic nonparenchymal macrophage, and are positively associated with the severity of liver fibrosis. They play an important role in the synthesis and deposition of the extracellular matrix by upregulating the expression of tissue inhibitor of metalloproteinases and downregulating the activity of matrix metalloproteinases. Cirrhosis, a chronic diffuse lesion usually accompanying extensive liver fibrosis and nodular regeneration, is caused by liver parenchymal cells repeating injury-repair following reconstruction of organizational structure in the hepatic lobules. Hepatocellular carcinoma is caused by repeated and persistent chronic severe liver injury, and partial hepatocytes can eventually transform into hepatoma cells. Multiple TLRs such as TLR2, TLR3, TLR4,
\end{abstract}


and TLR9, as well as other receptors, can be expressed in cirrhosis and hepatocellular carcinoma. About 53 and $85 \%$ of hepatocellular carcinoma patients frequently express TLR3 and TLR9, respectively. The chronic and repeated liver injury caused by alcohol, and HBV, $\mathrm{HCV}$, or other pathogens can be recognized by TLRs through the PAMP pathway, which directly increases the risk for hepatic cirrhosis and hepatocellular carcinoma. In this review, we briefly present evidence that the novel cellular molecular mechanisms of TLRs may provide more information about new therapeutics targets of the antiinflammatory immune response.

Key words: Toll-like receptor; Innate immunity; Hepatic cirrhosis; Hepatocellular carcinoma

\section{INTRODUCTION}

Toll-like receptors (TLRs), the major epithelial pattern recognition receptors (PRRs), are type I transmembrane receptors. They can specifically identify pathogen-associated molecular patterns (PAMPs) by recognizing structural patterns in diverse microbial molecules so that acquired immunity against microbial infectious through innate immunity can be established. The signal pathways of the TLRs in hepatocytes are similar to those in immune cells, and can be involved in the pathological processes of chronic hepatitis, cirrhosis, and hepatocellular carcinoma. PKR (protein kinase R) is an intermediary in the TLR signal transduction of doublestranded RNA (dsRNA)-dependent protein kinase. It participates in dsRNA-activated TLR3 signaling, and is recruited by a TAK1-containing complex in response to dsRNA binding to the TLR3 receptor (Carpentier et al., 2007). There is no direct evidence that the hepatitis B virus (HBV) (dsDNA) or the hepatitis C virus (HCV) (ssRNA) can directly stimulate the respective TLRs. Recent studies have confirmed that TLRs, which are cell surface receptor molecules, play an important role in the host antiviral immune response. They can identify viral genomes with high CpG (cytosine-phosphate-guanine) DNA content, dsRNA, and PAMPs, so that acquired immunity is activated on the basis of innate immunity producing multiple cytokines and upregulating the expression of co-stimulatory molecules (CD40, CD80, and CD86). The host TLRs can identify HBV, HCV, and other pathogenic microorganisms, and play an important role in the anti-inflammatory and anti-viral response via two major signal transduction cascades: the MyD88-dependent and MyD88-independent pathways.

The liver weighs approximately 1200-1500 g. It has a double blood supply, and constantly confronts intestinal bacterial components such as bacterial lipopolysaccharides (LPS) translocated from the gut into the portal vein, while maintaining its unique capacity to induce immune tolerance. It has been suggested that various TLRs can be widely expressed on hepatocytes, Kupffer cells, sinusoidal endothelial cells, and hepatic stellate cells (HSCs), and can maintain tolerance to repeated stimulation by intestinal bacterial antigens so as to prevent organ failure due to the overactivation of the immune system (Miyake and Yamamoto, 2013). In contrast, a breakdown in TLR tolerance may induce persistent inflammation, and contributes to the development of chronic liver diseases (Singh et al., 2011). In this review, we will initially focus on summing-up the structural characteristics of the extracellular domains 
and the transmembrane and intracellular signaling domains of the TLR family. We will then summarize recent discoveries concerning the molecular mechanism of TLR-activated innate immunity, and their contribution to our understanding of the adaptive immune responses to various inflammatory liver injuries in hepatic cirrhosis and hepatocellular carcinoma.

\section{Structural features and distribution characteristics of TLRs}

The discovery of the toll/interleukin-1 receptor (TIR) homology domain revealed the similarity between the intracellular domain of mammalian TLRs and that of interleukin-1 receptor (IL-1R). So far, 11 TLR family members have been identified in humans and 12 have been identified in mice. All TLRs are composed of extracellular, transmembrane, and intracellular signaling domains. Both humans and mice conserve TLR1-TLR9 and TLR14. Humans express TLR10 but mice do not (owing to a stop codon in the murine Tlr10 gene), whereas TLR11-TLR13 are expressed in mice but not in humans. The human TLRs can be divided into five subfamilies: TLR2, TLR3, TLR4, TLR5, and TLR9. Among them, the TLR2 subfamily consists of TLR1, TLR2, TLR6, and TLR10, and the TLR9 subfamily is composed of TLR7, TLR8, and TLR9. The TLR3, TLR4, and TLR5 families are only represented by one member each (Kumar et al., 2011). TLR1 and TLR6 have similar genomic structures, are arranged in series, contain a single exon, and have the same human chromosomal locus (4q14); the amino acid sequence homology between the proteins they encode reaches $69.3 \%$, and the TIR domain amino acid sequence homology is more than 90\% (Jang and Park, 2014). The TLR2 gene is located close by at the chromosomal locus 4q32; it contains two exons at the 5 ' coding end, and the coding sequences are located within exon 2. TLR3 has the human chromosomal locus 4q35; it has a special gene structure that includes five exons, but the amino acid sequence is coded by exons 2 to 5. TLR4 has the locus 9q32-33 and contains four exons. $T L R 5$ has the locus 1q33.3 and contains five exons. TLR 7 and TLR 8 have a common tandem arrangement in the X chromosome (locus Xp22) and contain two exons; their nucleotide sequence homology is up to $42.3 \%$ and the amino acid homology in the proteins they encode is as high as $72.7 \%$ (Mikula et al., 2010). TLR9 has the human chromosomal locus 3p21.3 and includes two exons; it is mainly expressed in the spleen, lymph nodes, bone marrow, and peripheral blood leukocytes. The activation of TLR9 can directly activate innate immunity via the NF- $\mathrm{KB}$ signaling pathway and is also involved in the cellular immune response (Chuang and Ulevitch, 2000). The TLR10 gene has the human chromosomal locus $4 \mathrm{p} 14$ and is mainly expressed in the lymphoid tissue and spleen cells; its protein is usually associated with the TLR1/2 heterodimer (Shinkai et al., 2006). TLR11, a recently discovered member of the TLR family, is mainly expressed in the urinary system. Mice with TLR11 deficiency are highly susceptible to bacterial infection of the kidneys; the total bacteria in the kidneys of mice with pyelonephritis are more than 1000 times that in normal mice. It has been proved that TLR11 plays an important role in the control of bacterial urinary tract infections.

TLRs are an important class of protein molecules and play a critical role in innate immune response; they are composed of an N-terminal extracellular domain (ECD), a transmembrane domain, and a C-terminal intracellular signaling domain. The extracellular domain has high sequence variability, has 18-28 leucine-rich repeat (LRR) modules sandwiched between the LRRNT and LRRCT modules, and contributes to specific binding to the corresponding ligands. The individual LRR module consists of 20-30 amino acids 
and is composed of conserved motifs and variable regions. The conserved "LxxLxLxxN" elements of the modules ( $x$ represents any amino acid, $L$ represents leucine) create the concave surfaces of the LRRs by forming parallel $\beta$-turns, while the variable parts of the modules form convex surfaces and consist of $\alpha$-helices and/or loops. The conserved region is the TIR domain, characteristic of the interleukin-1 receptor/toll-like receptor superfamily. The conserved sequence patterns in the LRR modules are usually regarded as unique horseshoeshaped entities and contribute to the recognition of various specific microbial PAMPs. The transmembrane domain of TLRs, which is a cysteine-rich domain, is generally believed to determine the subcellular localization of TLR molecules. The signaling cascade of TLRs can be divided into two main pathways depending on the intracellular adaptor molecules of recruitment: the MyD88-dependent signaling pathway and the MyD88-independent signaling pathway. The initial downstream adaptor molecules, including MyD88, TIRAP (TIRdomain-containing adaptor protein, also known as MyD88-adaptor-like protein, MAL), TRIF (TIR-domain-containing adaptor-inducing interferon- $\beta$ ), and TRAM (TRIF-related adaptor molecule), are required in the TLR signaling cascades. The dimerization of the intracellular signaling TIR domains can be enhanced by the dimerization of the extracellular domains, and the further recruitment of adaptor molecules triggers downstream signal transduction.

\section{Expression of TLRs in the liver}

The human liver, the largest human digestive gland, contains a large number of macrophages, such as Kupffer cells. Multiple TLRs can also be expressed in sinusoidal endothelial cells and HSCs, both of which are much more abundant in the liver. Various TLRs are expressed in the liver (Table 1). As the liver lies directly downstream of the gut in terms of anatomical location, it is constantly exposed to bacterial LPS from the intestines; it is regarded as the host's main filtration organ and plays an important role in the immune response. It has been confirmed that more than $80 \%$ of macrophages are present in the human liver, which is usually regarded as an important site for bacterial phagocytosis and clearance. Kupffer cells, the resident macrophages of the liver, are able to efficiently clean out bacterial endotoxins; they phagocytose bacteria through the portal vein, and play a key role in the clearance of systemic bacterial infections. Compared with other organs, the levels of TLR1, TLR2, TLR4, TLR6, TLR7, TLR8, TLR9, and TLR10 mRNA are low in healthy livers, which is also true for the mRNA levels of other signaling molecules such as MD-2 and MyD88. This suggests that the low expression of TLR signaling molecules might contribute to the high tolerance of the liver when constantly exposed to TLR ligands from intestinal bacterial LPS (Seki et al., 2011). The TLR3 and TLR5 mRNA levels are a little higher than those of the other TLRs in the liver, but they are still lower than those in the spleen. It is worth noting that the latest discoveries concerning the expression of TLRs widely expressing on hepatocytes and nonparenchymal cells are constantly being demonstrated (Wu et al., 2009); examples include: TLR1-9 in hepatocytes; TLR2-5 in HSCs; TLR2-4 in Kupffer cells; CD14, MD-2, and TLR2-5 in biliary epithelial cells; TLR2-4 and TLR9 in sinusoidal endothelial cells; and TLR2 and TLR4 in hepatic dendritic cells (DCs). Inflammatory and dsRNA viruses can directly induce enhanced cell apoptosis via the expression of the tumor necrosis factor-related apoptosis-inducing ligand (TRAIL). There is accumulating evidence that TLRs play an essential role in local immune defense against bacteria and viruses, and participate extensively in the pathological process of liver regeneration, fatty liver, and cirrhosis. 


\begin{tabular}{|c|c|c|c|}
\hline \multirow{4}{*}{$\begin{array}{l}\text { ILR subtamily } \\
\text { TL2 subfamily }\end{array}$} & Members & \begin{tabular}{|l} 
Expression (protein level) \\
\end{tabular} & \begin{tabular}{|l|l} 
Ligand (origin) \\
\end{tabular} \\
\hline & TLR1/2 & NK cells, DCs $(\mathrm{h})$ & Triacyl lipopeptides (bacteria and mycobacteria) \\
\hline & TLR2/6 & $\begin{array}{l}\text { Hepatocytes, Kupffer cells, NK cells, B cells, activated T cells, DCs (m), } \\
\text { biliary epithelial cells }\end{array}$ & $\begin{array}{l}\text { Lipoprotein/lipopeptides (various pathogens), peptidoglycan, lipoteichoic acid ( } \mathrm{G}^{+} \\
\text {bacteria), lipoarabinomannan (mycobacteria), zymosan (fungi) }\end{array}$ \\
\hline & TLR10 & Unknown & ND \\
\hline TLR3 subfamily & TLR3 & $\begin{array}{l}\text { Hepatocytes, LSECs, Kupffer cells, NK cells, NKT cells, activated T cells, } \\
\text { cDCs (m), biliary epithelial cells }\end{array}$ & Double-stranded RNA (viruses) \\
\hline TLR4 subfamily & TLR4 & $\begin{array}{l}\text { Hepatocytes, LSEC, Kupffer cells, NK cells, B cells, activated T cells, DCs } \\
\text { (m), biliary epithelial cells, HSCs }\end{array}$ & $\begin{array}{l}\text { Lipopolysaccharide (G- bacteria), Fusion protein (Respiratory syncytial virus), envelope } \\
\text { protein (mouse mammary-tumor virus); HMGB1, hyaluronan, HSP60, free fatty acids } \\
\text { (endogenous ligands); HSP72 (cells during stress and injury) }\end{array}$ \\
\hline \multirow{4}{*}{$\begin{array}{l}\text { TLR9 subfamily } \\
\text { TLR }\end{array}$} & TLR5 & Biliary epithelial cells & Flagellin (bacteria) \\
\hline & TLR7/8 & NK cells, B cells, DCs $(\mathrm{h}), \mathrm{DCs}(\mathrm{m})$ & Single-stranded RNA (viruses), double-stranded, short-interfering RNA (siRNA) \\
\hline & TLR9 & $\begin{array}{l}\text { LSECS, Kupffer cells, NK cells, B mDCs and macrophages cells, activated T } \\
\text { cells, pDCs }(\mathrm{h}), \mathrm{DCs}(\mathrm{m}), \mathrm{HSCs}\end{array}$ & \begin{tabular}{|l|l|} 
Unmethylated CpG motifs of DNA (bacteria and viruses) \\
\end{tabular} \\
\hline & TLR11 & Kupffer cells & \begin{tabular}{|l|l} 
Profilin-like molecule (protozoa) \\
\end{tabular} \\
\hline
\end{tabular}

Hepatocytes, which are parenchymal cells, account for $60-89 \%$ of the total cell population in the liver. They are the major source of PRR production, including secreted PRRs and membrane-bound PRRs. In healthy livers, the low expression of TLRs may contribute to the innate tolerance of the liver to TLR ligands from intestinal microbes. Under the stimulation of inflammatory factors, the level of TLR2 in hepatocytes is upregulated and hepatocytes become more sensitive to TLR2 ligands. However, the expression of TLR4 in hepatocytes is usually not upregulated. Among the various nonparenchymal cells in the healthy liver, liver sinusoidal endothelial cells (LSECs) are the most numerous, and account for more than $50 \%$. The liver can express TLR3, TLR4, and TLR9 to promote multiple pathogenic agents or eliminate cellular debris. Wu et al. (2010) found that TLR2, -4, and -6 agonists stimulated Kupffer cells, and TLR1, -2 , and -6 agonists stimulated LSEC-induced T-cell proliferation, as assessed by IFN- $\gamma$ production and proliferative activity. Interestingly, under the stimulation of TLR1 and TLR2 in particular, high levels of IFN- $\gamma$ expression by Kupffer cells and LSECs could be induced at Days 2 and 3, which was comparable with the observations of mixed lymphocyte reactions in myeloid dendritic cells ( $\mathrm{mDCs})$.

\section{Expression of TLRs in liver fibrosis}

Liver fibrosis is a pathological process that occurs without pseudo-lobule formation and is caused by multiple pathogenic factors, such as large hepatocyte damage, inflammatory cell aggregation, activation, and proliferation of liver myofibroblasts, and extracellular matrix (ECM) oversecretion and accumulation. Multiple TLRs are widely expressed in the liver on Kupffer cells, the sinusoidal endothelium, dendritic cells, biliary epithelial cells, HSCs, and hepatocytes. There is increasing evidence for the participation of TLRs in the activation of hepatic immune cells and stellate cells during liver fibrosis. Inflammation, regarded as one of important factors, is strongly associated with chronic hepatic injury and the ensuing wound-healing process. It increases the initiation and promotion of liver fibrosis and drives the activation of HSCs; activated HSCs transdifferentiate into myofibroblasts, which then produce excessive quantities of ECM proteins (Wang et al., 2009). A large number of studies have shown that the endotoxin levels in healthy individuals are less than $3 \mathrm{pg} / \mathrm{mL}$, while the endotoxin levels are up to $4.9,7.9$, and $10.2 \mathrm{pg} / \mathrm{mL}$ in the Child-Pugh classes A, B, C, respectively, in patients with liver fibrosis in vivo (Lata et al., 2007).

HSCs are mainly found in the perisinusoidal space (also known as the space of Disse) and are in direct contact with hepatocytes and sinusoidal endothelial cells; they are regarded as playing a key role in the development of liver fibrosis after activation and proliferation. Human 
activated HSCs can express TLR4 and CD14, and also identify LPSs in the cell walls of Gramnegative bacteria. They are actively involved in liver fibrosis via secretion of extracellular matrix products. TLR4 acts as a receptor for LPS and mediates its intracellular actions via the downstream key adapter molecule MyD88. It is noteworthy that TRL4-dependent signaling is an important mediator of liver fibrosis, as has been demonstrated in experiments using a knockout mouse model of MyD88. In addition, previous studies in animals have suggested that TLR2, TLR3, and TLR9 are also involved in the development and progression of liver fibrosis. Gäbele et al. (2008) explored the effects on liver fibrosis of TLR9 expressed by HSCs activated by $\mathrm{CpG}$ motifs present specifically in bacterial DNA. When subjected to CpG stimulation, the levels of the profibrogenic chemokine monocyte chemotactic protein 1 (MCP1) quickly increased in human as well as murine HSCs isolated from wild-type (TLR9+/+) mice. In contrast, HSCs isolated from TLR9-deficient (TLR9-/-) mice lacked CpG motifinduced MCP-1 expression, indicating the functionality of TLR9 in HSCs. The levels of hepatic MCP-1 and collagen expression and liver fibrosis in TLR9-/- mice were significantly lower than in TLR9+/+ mice after bile duct ligation. Therefore, the CpG motif in bacterial DNA can induce the activation of HSCs and further promote liver fibrosis.

LPS, an important TLR4 ligand and the main component of the cell walls of Gramnegative bacteria, provokes a strong immune response. CD14, an auxiliary LPS recognition receptor, is anchored to the membrane by a glycosylphosphatidylinositol tail, and improves TLR4 signaling activation by helping TLR4 identify LPS so that sensitivity to LPS is increased. Intestinal microflora constitute more than $99 \%$ of the bacterial mass in the body and are the principal source of bacterially derived PAMPs in healthy as well as in many disease states. Such gut flora can enhance bacterial clearance against Escherichia coli pneumonia through the TLR4 pathway. The intestinal microflora-derived LPS translocates into the liver via the portal vein by increased intestinal permeability following liver injury. The stimulation of TLR4 leads to the production of various cytokines (such as IL-1, IL-6, and TNF- $\alpha$ ) and chemokines (such as MCP-1, MIP-1 $\beta$, and RANTES) in HSCs, and recruits Kupffer cells through their CCR1 and CCR2. In addition, Kupffer cells can mediate the expression of a variety of cytokines and chemokines in the liver after LPS injection. LPS also directly targets quiescent HSCs via downregulation of the TGF- $\beta$ pseudoreceptor BAMBI (also known as BMP and activin membrane-bound inhibitor homolog). The expression of BAMBI is downregulated in response to LPS and TNF- $\alpha$ in human HSCs. LPS stimulation can also downregulate the expression of BAMBI in HSCs with NF- $\mathrm{BB} / \mathrm{p} 65$ knockdown. LPS- and TNF- $\alpha$-induced NF$\kappa \mathrm{B}$ interaction represses BAMBI transcriptional activity, which could contribute to TLR4mediated enhancement of TGF- $\beta$ signaling in HSCs during liver fibrosis (Liu et al., 2014). A large quantity of ECM material is secreted from activated HSCs and contributes to liver fibrosis progression. Thus, TLR4 may play a critical role in liver fibrosis (Figure 1).

Kupffer cells, which are special nonparenchymal hepatic macrophages and constitute the largest macrophage group in the body, account for $80-90 \%$ of the monocyte-macrophage cell system, $15 \%$ of the total number of hepatocytes, and $30 \%$ of the total number of sinusoidal endothelium cells (Molawi and Sieweke, 2015). TLR4 can be widely expressed in the monocyte-macrophage system and is involved in the regulation of target gene transcription in liver fibrosis via the TLR4-NF- $\kappa$ B and TLR4-MAPK signaling pathways, and the release of various inflammatory mediators and cytokines. It has been shown that the levels of IL-1 resulting from autocrine action in fibroblasts and paracrine action in Kupffer cells are 
positively associated with the severity of liver fibrosis. IL-1 also plays an important role in the synthesis and deposition of ECM materials through the upregulation of tissue inhibitor of metalloproteinases (TIMP) and the downregulation of matrix metalloproteinases (MMPs) (Mòdol et al., 2015). Kupffer cells also have multiple biological effects on hepatocytes, HSCs, and endothelial cells. Seki et al. (2007) and other researchers found that the nuclei in a fresh isolate of HSCs had low levels of NF- $\mathrm{KB}$, but the nuclei of activated HSCs had high levels of NF- $\mathrm{KB}$, which was accompanied by the expression of the intercellular adhesion molecule, VCAM-1 and by the expression of other genes. It has also been suggested that HSCs can activate target gene transcription and release a series of cytokines through MyD88-NF- $\mathrm{BB}$ signaling transduction, and ultimately may induce HSCs activation and fibrosis.

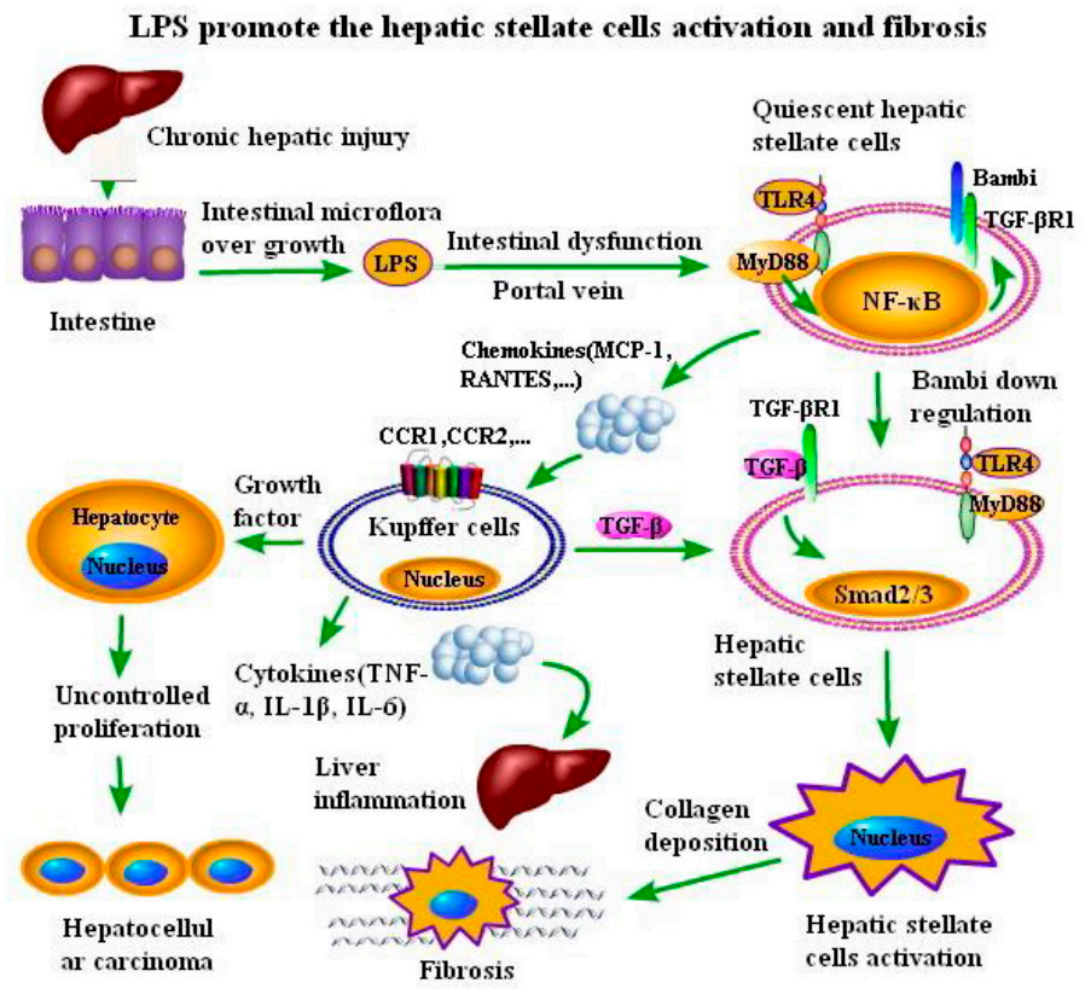

Figure 1. Lipopolysaccharides (LPSs) induce hepatic stellate cell activation and fibrosis. LPSs produced by intestinal bacteria via the portal vein directly target quiescent hepatic stellate cells, and the release of TGF-b1 from Kupffer cells against the hepatic stellate cells can gradually induce events such as hepatic stellate cell activation, collagen deposition, and liver fibrosis. Kupffer cells produce a variety of pro-inflammatory factors that induce inflammatory liver injury. A variety of chemokines produced by hepatic stellate cells following stimulation of TLR4 have an effect on Kupffer cells, and hepatocellular carcinoma may easily be induced by persistent hepatocyte injury and uncontrolled proliferation.

\section{Role of Kupfer cells in the relationship between TLRs and hepatic cirrhosis or hepatocellular carcinoma}

In general, normal livers contain low levels of the mRNAs of TLRs and those of their 
downstream signaling molecules, such as MyD88, TRIF, and NF- $\mathrm{BB}$, compared with other organs. However, the overgrowth of intestinal bacteria and increased intestinal permeability are positively related to the development of cirrhosis. Multiple antigen exposure and recognition via TLRs in the liver can trigger some distinctive mechanisms, and are further conducive to the maintenance of self-tolerance and anti-infection immunity. Kupffer cells within the liver can express multiple TLRs, such as TLR2, TLR3, TLR4, and TLR9, and frequently participate in phagocytosis and antigen presentation to remove gut-derived endotoxins (such as LPS). They are particularly sensitive to gut-derived endotoxins, acting through CD14, TLR2, TLR4, and adapter proteins such as MD2 to activate NF- $\mathrm{KB}$ via the MyD88 pathway. Interestingly, the pathological effect of TLR4 in Kupffer cells is achieved by inducing reactive oxygen species (ROS)-dependent activation of X-box binding protein-1 (XBP-1) in mediating the transition from steatosis to inflammation (Ye et al., 2012). The ApoE(-/-)/TLR4(mut) mice lacked functional TLR4 and were resistant to high-fat, high-cholesterol (HFHC) diet-induced liver inflammation and injury, and were less susceptible to the diet-induced production of ROS and proinflammatory cytokines. It has been suggested that the TLR4 in Kupffer cells plays a critical role in mediating the progression of simple steatosis to nonalcoholic steatohepatitis via ROS-dependent activation of XBP-1.

Kupffer cells are putative liver tissue macrophages and can express most of the major TLRs. It seems clear that TLRs play a role in the development of inflammation-associated hepatocellular carcinoma. The activated TLR4 on HSCs could promote recruitment of Kupffer cells and directly augment the fibrogenic response through downregulation of BAMBI. The dead hepatocytes might further promote the activation of Kupffer cells via TLRs and induce production of inflammatory cytokines and mitogen in the development of hepatocellular carcinoma. The data provided by Naugler et al. (2007) showed that exposure to the chemical carcinogen diethylnitrosamine promoted production of IL-6 in Kupffer cells in a manner that was dependent on the toll-like receptor adaptor protein MyD88 in the generation of hepatocellular carcinoma. Mice deficient in TLR4 and MyD88, but not in TLR2, showed notable decreases in the incidence and size of chemically induced hepatocellular carcinomas.

\section{Expression of TLRs in hepatic cirrhosis}

Cirrhosis, a chronic diffuse lesion usually accompanying extensive liver fibrosis and nodular regeneration, is caused by liver parenchymal cells repeating injury-repair following reconstruction of organizational structure in the hepatic lobules. It is now recognized as essentially late-stage fibrosis that is triggered by repeated injury-inflammation caused by factors including chronic hepatitis B and C, autoimmune hepatitis, nonalcoholic steatohepatitis, and alcoholic liver disease.

Peripheral blood mononuclear cells (PBMCs) have multiple activated immune cells, such as T lymphocytes, monocytes, macrophages, and dendritic cells, and play an important role in the immune response to foreign pathogens (Wang and Lu, 2008; Wang et al., 2008, 2010). The expression of TLR 4 and TLR2 in PBMCs, circulating TNF- $\alpha$ levels, and TNF- $\alpha$ production were investigated in 36 cirrhotic patients in an early study by Riordan et al. (2003). The levels of TLR2 but not TLR4 in PBMCs were significantly upregulated in cirrhosis and significantly correlated with the levels of TNF- $\alpha$ and soluble TNF receptor (sTNFR) in the serum. Supplementation with the symbiotic regimen resulted in significant upregulation of 
TLR2 in PBMCs. The expression of TLR2 might contribute to high levels of circulating TNF- $\alpha$ and sTNFR levels in cirrhosis patients.

The pathway of pro-/anti-inflammatory cytokine release can be activated by any increase in pathological bacterial translocation (BT) products to the gut-associated lymphoid tissue. Most BT products comprise lipopolysaccharides, peptidoglycans, muramyl-dipeptides, and bacterial DNA. Interestingly, many clinical trials have reported that pathological intestinal BT develops with a sustained increase in the rate and/or degree of cirrhosis, and can further lead to a change in the status of cirrhosis from compensated to decompensated (Figure 2). Usually, anaerobes outnumber aerobes by 100:1 and limit the colonization and overgrowth of other potentially invasive microbes, so that multiple potentially pathogenic bacteria are restricted. However, the common bacteria that translocate most readily are facultative intracellular pathogens or opportunistic pathogens, such as Salmonella species, E. coli, Klebsiella pneumoniae, and other Gram-negative bacteria, and can be resistant to phagocytosis. It is noteworthy that $E$. coli, the most common pathogenic bacterium, can frequently cause spontaneous bacterial peritonitis (SBP) in cirrhosis. For example, Sheikhbahaei et al. (2014) reported that $24.33 \%$ of the samples they studied were diagnosed with SBP, from which $65.15 \%$ were culture-positive. The most prevalent isolated pathogen was E. coli (33.8\%), followed by Staphylococcus aureus $(8.9 \%)$ and Enterococcus (8.6\%). It was surprising that the percentage of E. coli strains that were resistant to cefotaxime, ceftazidime, and ciprofloxacin increased significantly to $72.7 \%, 75.8 \%$, and $54.3 \%$. The pathological intestinal BT was not conducive to recovery from liver cirrhosis.

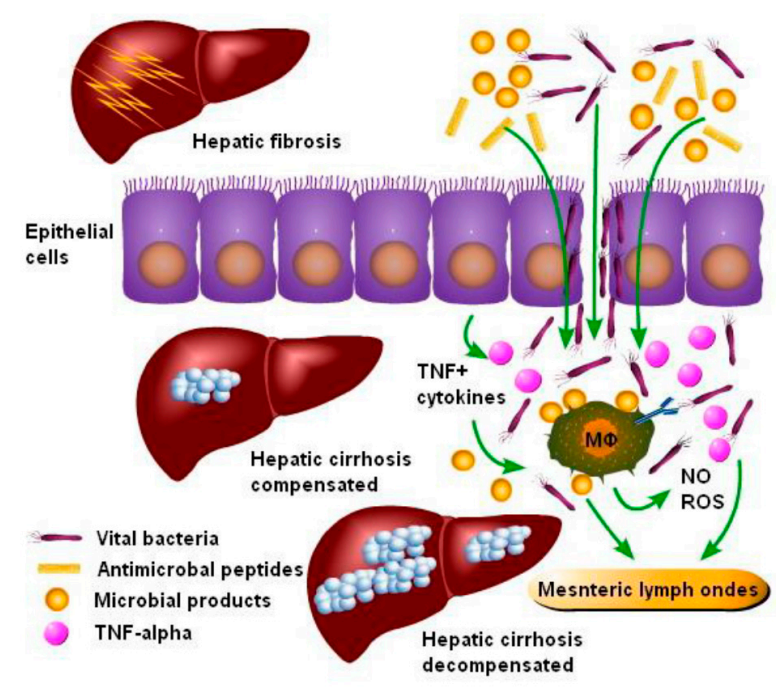

Pathological bacterial translocation and cirrhosis

Figure 2. Pathological bacterial translocation (BT) and development of cirrhosis.

In cases of hepatic cirrhosis with ascites, there is excessive growth of intestinal bacteria due to a state of tolerance in the intestinal epithelial cells. This can enhance pathogenic over-transcytosis of viable bacteria in the gut-associated lymphoid tissue, and further lead to a vicious circle perpetuating BT through a relative absence of bacterial destruction. TNFsecretion is likewise increased in the mesenteric lymph nodes (MLN) and serum in cases of 
human cirrhosis with ascites. Reactive oxygen species (ROS) can increase the internalization rate of Escherichia coli into epithelial cells and enhance adhesion via the pathway of modulating brush border glycosylation. Overproduction of nitric oxide (NO) is deleterious to the integrity of the intestinal epithelium.

ROS are biological byproducts of the process of oxygen metabolism, and include oxygen ions, peroxides, and oxygen free radicals. They can increase the rate of internalization of $E$. coli into epithelial cells in vitro and enhance the adhesion of bacteria via the pathway of modulating brush border glycosylation. Bruns et al. (2011) showed that the fraction of neutrophils spontaneously producing ROS increased in cases of liver cirrhosis. The levels of ROS and lipopolysaccharide-binding protein (LBP) increased with Child-Pugh stage in resting neutrophils, and positively correlated with the release of ROS in response to opsonized $E$. coli in the patients with cirrhosis. It has been demonstrated that the respiratory burst increase was positively associated with the severity of liver cirrhosis, and was restrained by the concentrations of LBP. Interestingly, the secretion of TNF- $\alpha$ by activated monocytes might increase resting burst in neutrophils.

Nitric oxide (NO), which is a small and important signaling molecule, can penetrate any cell, and plays an important role in modulating macrophage function, cytokine release, and bactericidal capacity, as well as maintaining intestinal barrier function. However, overproduction of $\mathrm{NO}$ in the splanchnic circulation is deleterious to the integrity of the intestinal epithelium. High concentrations of $\mathrm{NO}$ can directly induce gastric mucosal damage and decrease the viability of intestinal epithelial cells (Sohn et al., 2012), and may directly open the tight junctions in the intestinal epithelial monolayers; loosening of the tight junctions may result in increased accessibility of bacterial products to areas of "free" passage in cases of cirrhosis. Subsequently, ATP-formation can be inhibited and intestinal permeability increased.

Nitric oxide synthases (NOSs), including constitutive nitric oxide synthase (cNOS) and inducible nitric oxide synthase (iNOS), are produced by endothelial cells, nerve cells, and macrophages, and contribute to the catalytic synthesis of NO. Resting cells do not usually express iNOS. However, under the inducement of cytokines or microbial components (such as LPS), the catalyzed synthesis of large non-physiological concentrations of NO occurs with subsequent pathological stress responses. Many experiments have provided evidence that iNOS-derived NO can play an important role in promoting pathogenic BT in endotoxemia, hemorrhagic shock, and hepatic cirrhosis. The overproduction of NO results in the release of activated intestinal macrophages in decompensated cirrhosis, and may decrease transepithelial resistance and disrupt intestinal barrier function (Du Plessis et al., 2013). Similar results have also been confirmed in iNOS knockout mice exposed to LPS, which exhibited reduced mortality and absent BT.

Nucleotide-binding oligomerization domain-containing protein 2 (NOD2) exists in the cytoplasm and is an important member of the cytosolic pattern recognition receptors (PRRs) family, which have highly conserved gene sequences; it is widely distributed in plants, nematodes, vertebrates, and human cells. NOD2 is highly expressed in monocytes and Paneth cells in humans, and recognizes muramyl dipeptide (MDP), a pyrolysis product of the peptidoglycan (PNG) present in the bacterial wall of Gram-positive and Gram-negative bacteria. After recognizing the corresponding ligand, NOD2 can induce the release of pro-inflammatory cytokines and chemokines via the NF- $\mathrm{\kappa B}$ and MAPK cascade of signal transduction. TLR2 is widely expressed on the cell surface of macrophages and other immune- 
competent cells so as to promptly recognize the PAMPs of Gram-positive organisms. TLR2deficient mice present with obviously attenuated BT from the intestine to the mesenteric lymph nodes and endotoxinemia (Hartmann et al., 2012). In a recent study by Nischalke et al. (2011), TLR2 and intron 2 polymorphisms were reported to be associated with increased risk for spontaneous bacterial peritonitis in hepatic cirrhosis. The data from the hybridization probe assays using a LightCycler system indicated that TLR2 polymorphisms increased supplementary risk factors for SBP in cirrhosis with ascites, and the combination of the TLR2 GT microsatellite polymorphism with at least one NOD2 risk variant enables improved identification of patients at high risk of SBP. The study underlined the interaction between NOD2 and TLRs, particularly in the modulation of TLR2-dependent cytokine responses. Therefore, alterations in NOD2- and TLR2-function at different cellular sites must play a key role in susceptibility to pathological BT.

More advanced pathological changes, such as weak clearing capacity of Kupffer cells, intestinal bacterial translocation and overgrowth, and endotoxemia, are normally seen in massive necrosis of hepatocytes and gastrointestinal mucosa congestion in patients with cirrhosis. Shimoda et al. (2011) successfully isolated subpopulations of liver mononuclear cells and examined the role of TLRs and their ligands, and natural killer (NK) cells in the participation of cytotoxic activity against biliary epithelial cells. The data confirmed and highlighted that there were critical differences were present in the varied roles of monocytes and NK cells following TLR3-L and TLR4-L stimulation. Zhao et al. (2011) also determined the levels of LPS and pro-inflammatory cytokines in serum, and the expression of LPS receptors in liver biopsy tissues from 162 patients with primary biliary cirrhosis. The results also showed that significantly higher levels of LPS were detected in patients with primary biliary cirrhosis in comparison with patients with other liver diseases and with healthy controls, and high expression levels of TLR4, CD14, CD68, and NF- $\kappa B$ were investigated in liver biopsy by immunohistochemical staining. After LPS stimulation in vitro, the expression of TLR4 and pro-inflammatory cytokine increased significantly in monocytes with positive CD14 expression and in cultured human biliary epithelial cells from patients with primary biliary cirrhosis. It has been confirmed that TLR4 can recognize lipopolysaccharides from Gram-negative bacteria. However, further research by Morita et al. (2013) was carried out on TLR4 single-nucleotide polymorphisms in 261 patients with primary biliary cirrhosis (PBC) and 359 healthy subjects. The results showed that TLR4 polymorphisms did not play a prominent role in the development of $\mathrm{PBC}$ in Japanese patients, and there was no significant association between TLR4 single-nucleotide polymorphisms or haplotypes and late-stage PBC or cirrhosis.

\section{Expression of TLRs in hepatocellular carcinoma (HCC)}

HCC is a common malignant tumor and the 6th most likely cause of cancer death worldwide. It involves the expression of multiple TLRs such as TLR2, TLR3, TLR4, and TLR9. Approximately $53 \%$ and $85 \%$ of HCC patients express TLR3 and TLR9, respectively. Both TLR3 and TLR9 can be expressed on the surface of cell membranes and in the cytoplasm of hepatoma cell lines (Eiró et al., 2014). TLR9 agonists induce upregulation of anti-apoptosis genes and promote cancer cell proliferation. The chronic and repeated liver injury caused by alcohol, HBV, HCV, and other pathogens can be recognized by TLRs through the PAMPs 
pathway, which directly increase the risk for hepatocellular carcinoma. There is increasing evidence that TLRs as well as other critical regulators of cytokines such as NF- $\mathrm{\kappa B}$ may promote liver tumorigenesis (Mencin et al., 2009). However, it is highly probable that fibrosis-associated hepatocellular carcinoma can be mediated by TLR signaling, because TLR4 and MyD88 are required for the development of hepatocellular carcinoma. A key role of the TLR signaling molecule MyD88 is the mediation of IL-1R production, as shown by diethylnitrosamineinduced hepatocellular carcinoma in male mice (Naugler et al., 2007), but this tumor-promoting pathway may be mediated by IL-1R or another upstream activator of MyD88. Therefore, it is possible that hepatocellular carcinomas associated with diethylnitrosamine-induced fibrosis and non-fibrosis may be MyD88-dependent but could also be mediated by different signaling cascades. To accelerate HCC formation in NS5A transgenic mice, Machida et al. (2009) took advantage of the upregulation of the TLR4 signal transduction pathway induced by ethanol. It was also shown that NS5A transgenic (NS5A Tg) mouse livers overexpressed TLR4, but ethanol and LPS feeding increased TLR4 signal transduction due to the endotoxemia caused by ethanol. NS5A transgenic mice easily overreacted to LPS with the development of massive hemorrhage, necrosis, and inflammation. Thirty percent of the NS5A Tg mice were killed by LPS challenge, but that was not the case in the wild-type mice or the NS5A Tg/TLR4-/- mice.

To gain insight into the role of TLR7 in the pathogenesis of HCC, 87 specimens of cancerous and noncancerous liver tissue were investigated by immunohistochemical staining and quantitative polymerase chain reaction (Lin et al., 2013). The data showed that higher expression of TLR7 was found in normal regions compared with the hepatocytes of cancerous regions in HCC. The levels of TLR7 in the adjacent normal liver tissue were also higher than in the tumor tissue (such as HBV-related HCC and HCV-related HCC). A significant difference in TLR7 mRNA expression was found between normal and cancerous tissues of $\mathrm{HCV}$-related HCC, and little significant difference was observed between HBV-infected and HCV-infected HCC. These data indicate that TLR7 is downregulated in HCV-related HCC. However, Mohamed et al. (2015) reported different results. Their data, produced by immunohistochemistry staining, showed that TLR7 and TLR9 were both overexpressed in the HCC tissues compared with those from cirrhosis and viral hepatitis cases. The proliferation of $\mathrm{HuH} 7$ cells was significantly increased after stimulation with the TLR7 agonist IMQ in vitro, but was not significantly increased in response to stimulation with the TLR7 agonist CpG ODN. TLR7 and TLR9 inhibition using IRS-954 or chloroquine significantly reduced $\mathrm{HuH7}$ cell proliferation in vitro and inhibited tumor growth in a mouse xenograft model. The proliferation of $\mathrm{HuH} 7$ cells was inhibited by a TLR7 inhibitor (chloroquine, CQ) in vitro, and tumor growth was inhibited by the TLR9 inhibitor IRS-954 in the mouse xenograft model. This indicates that the inhibition of TLR7 and TLR9 with IRS-954 or chloroquine might potentially be used as a novel therapeutic approach for preventing HCC development and/or progression in susceptible patients. An exciting report from Zhang et al. (2014) showed that phosphorothioate-modified $\mathrm{CpG}$ oligodeoxynucleotide (CpG ODN) induced apoptosis via the TLR9 pathway in human hepatocellular carcinoma cells. HepG2 and $\mathrm{H} 7402$ cells from human HCC cell lines were transfected with CpG ODNs (TLR9 agonist ODN M362 and its negative control ODN M362 ctrl) or small interfering RNAs (siRNAs) targeting TLR9. Interestingly, similar proapoptotic effects of ODN M362 and ODN M362 Ctrl were found in HCC, and might be related to phosphorothioate-modified CpG ODN. These findings suggest that the phosphorothioate-modified TLR9 agonist ODN M362 can induce positive antitumor activity 
in $\mathrm{HCC}$, and may be regarded as a novel therapeutic target for HCC therapy.

In summary, we discussed the details of the structure and function of the signal transduction pathway of TLRs, and we initially clarified that TLRs recognize HBV, HCV, and other pathogens in their conservative region through the PAMP pathway and the activation of downstream molecules such as MyD88 in innate immunity. TLRs can induce a variety of pathogen-related cytokines, chemokines, and type I interferons released from host $\mathrm{T}$ cells and $\mathrm{B}$ cells, and upregulate the expression of co-stimulatory molecules in acquired immunity. Understanding the novel cellular molecular mechanisms of TLRs will be useful in providing more information about possible new therapeutic targets for the regulation of TLR activity in anti-virus and anti-inflammatory immune response. Hepatitis viruses (HBV and HCV) have multiple ligands, which may activate multiple signaling pathways, and lead to crosstalk between the various signaling cascades, resulting in a wide range of innate immune responses. To date, studies have shown that TLRs, RIG-I-like receptors (RLRs), and other sensors are not sufficient to mount protective immune responses against a wide range of viral infections, and more unidentified PRRs may play a key role in defense against an array of viral pathogens. Therefore, the identification and characterization of new TLRs and PRRs is necessary, and host-pathogen interactions and the development of various therapeutic strategies are worthy of further study.

\section{Conflicts of interest}

The authors declare no conflict of interest.

\section{ACKNOWLEDGMENTS}

Research supported by grants from the Science Foundation of Anhui Province, China (\#1308085MH148; \#090413138) and the Educational Science Foundation of Anhui Province, China (KJ2010A086). Research also supported by key grants from the Educational Science Foundation of Anhui Province, China (\#KJ2016ZD20) and the science projects of China Coal Industry Association (\#MTKJ 2015-333).

\section{REFERENCES}

Bruns T, Peter J, Hagel S, Herrmann A, et al. (2011). The augmented neutrophil respiratory burst in response to Escherichia coli is reduced in liver cirrhosis during infection. Clin. Exp. Immunol. 164: 346-356. http://dx.doi.org/10.1111/j.1365$\underline{2249.2011 .04373 . x}$

Carpentier PA, Williams BR and Miller SD (2007). Distinct roles of protein kinase R and toll-like receptor 3 in the activation of astrocytes by viral stimuli. Glia 55: 239-252. http://dx.doi.org/10.1002/glia.20450

Chuang TH and Ulevitch RJ (2000). Cloning and characterization of a sub-family of human toll-like receptors: hTLR7, hTLR8 and hTLR9. Eur. Cytokine Netw. 11: 372-378.

Du Plessis J, Vanheel H, Janssen CE, Roos L, et al. (2013). Activated intestinal macrophages in patients with cirrhosis release NO and IL-6 that may disrupt intestinal barrier function. J. Hepatol. 58: 1125-1132. http://dx.doi. org/10.1016/j.jhep.2013.01.038

Eiró N, Altadill A, Juárez LM, Rodríguez M, et al. (2014). Toll-like receptors 3, 4 and 9 in hepatocellular carcinoma: Relationship with clinicopathological characteristics and prognosis. Hepatol. Res. 44: 769-778. http://dx.doi. org $/ 10.1111 /$ hepr.12180

Gäbele E, Mühlbauer M, Dorn C, Weiss TS, et al. (2008). Role of TLR9 in hepatic stellate cells and experimental liver fibrosis. Biochem. Biophys. Res. Commun. 376: 271-276. http://dx.doi.org/10.1016/j.bbrc.2008.08.096 
Hartmann P, Haimerl M, Mazagova M, Brenner DA, et al. (2012). Toll-like receptor 2-mediated intestinal injury and enteric tumor necrosis factor receptor I contribute to liver fibrosis in mice. Gastroenterology 143: 1330-40.e1. http:// dx.doi.org/10.1053/j.gastro.2012.07.099

Jang TH and Park HH (2014). Crystal structure of TIR domain of TLR6 reveals novel dimeric interface of TIR-TIR interaction for toll-like receptor signaling pathway. J. Mol. Biol. 426: 3305-3313. http://dx.doi.org/10.1016/j.jmb.2014.07.024

Kumar H, Kawai T and Akira S (2011). Pathogen recognition by the innate immune system. Int. Rev. Immunol. 30: 16-34. http://dx.doi.org/10.3109/08830185.2010.529976

Lata J, Novotný I, Príbramská V, Juránková J, et al. (2007). The effect of probiotics on gut flora, level of endotoxin and Child-Pugh score in cirrhotic patients: results of a double-blind randomized study. Eur. J. Gastroenterol. Hepatol. 19: 1111-1113. http://dx.doi.org/10.1097/MEG.0b013e3282efa40e

Lin KJ, Lin TM, Wang CH, Liu HC, et al. (2013). Down-regulation of Toll-like receptor 7 expression in hepatitis-virusrelated human hepatocellular carcinoma. Hum. Pathol. 44: 534-541. http://dx.doi.org/10.1016/j.humpath.2012.06.015

Liu C, Chen X, Yang L, Kisseleva T, et al. (2014). Transcriptional repression of the transforming growth factor $\beta$ (TGF- $\beta$ ) Pseudoreceptor BMP and activin membrane-bound inhibitor (BAMBI) by Nuclear Factor $\kappa B(N F-\kappa B)$ p 50 enhances TGF- $\beta$ signaling in hepatic stellate cells. J. Biol. Chem. 289: 7082-7091. http://dx.doi.org/10.1074/jbc.M113.543769

Machida K, Tsukamoto H, Mkrtchyan H, Duan L, et al. (2009). Toll-like receptor 4 mediates synergism between alcohol and HCV in hepatic oncogenesis involving stem cell marker Nanog. Proc. Natl. Acad. Sci. USA 106: 1548-1553. http://dx.doi.org/10.1073/pnas.0807390106

Mencin A, Kluwe J and Schwabe RF (2009). Toll-like receptors as targets in chronic liver diseases. Gut 58: 704-720. http://dx.doi.org/10.1136/gut.2008.156307

Mikula I, Bhide M, Pastorekova S and Mikula I (2010). Characterization of ovine TLR7 and TLR8 protein coding regions, detection of mutations and Maedi Visna virus infection. Vet. Immunol. Immunopathol. 138: 51-59. http://dx.doi. org/10.1016/j.vetimm.2010.06.015

Miyake Y and Yamamoto K (2013). Role of gut microbiota in liver diseases. Hepatol. Res. 43: 139-146. http://dx.doi. org/10.1111/j.1872-034X.2012.01088.x

Mòdol T, Brice N, Ruiz de Galarreta M, García Garzón A, et al. (2015). Fibronectin peptides as potential regulators of hepatic fibrosis through apoptosis of hepatic stellate cells. J. Cell. Physiol. 230: 546-553. http://dx.doi.org/10.1002/ jcp. 24714

Mohamed FE, Al-Jehani RM, Minogue SS, Andreola F, et al. (2015). Effect of toll-like receptor 7 and 9 targeted therapy to prevent the development of hepatocellular carcinoma. Liver Int. 35: 1063-1076. http://dx.doi.org/10.1111/liv.12626

Molawi K and Sieweke MH (2015). Monocytes compensate Kupffer cell loss during bacterial infection. Immunity 42: 1012. http://dx.doi.org/10.1016/j.immuni.2014.12.032

Morita S, Joshita S, Umemura T, Katsuyama Y, et al. (2013). Association analysis of toll-like receptor 4 polymorphisms in Japanese primary biliary cirrhosis. Hum. Immunol. 74: 219-222. http://dx.doi.org/10.1016/j.humimm.2012.10.022

Naugler WE, Sakurai T, Kim S, Maeda S, et al. (2007). Gender disparity in liver cancer due to sex differences in MyD88dependent IL-6 production. Science 317: 121-124. http://dx.doi.org/10.1126/science.1140485

Nischalke HD, Berger C, Aldenhoff K, Thyssen L, et al. (2011). Toll-like receptor (TLR) 2 promoter and intron 2 polymorphisms are associated with increased risk for spontaneous bacterial peritonitis in liver cirrhosis. J. Hepatol. 55: 1010-1016. http://dx.doi.org/10.1016/j.jhep.2011.02.022

Riordan SM, Skinner N, Nagree A, McCallum H, et al. (2003). Peripheral blood mononuclear cell expression of tolllike receptors and relation to cytokine levels in cirrhosis. Hepatology 37: 1154-1164. http://dx.doi.org/10.1053/

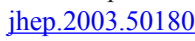

Seki E, De Minicis S, Osterreicher CH, Kluwe J, et al. (2007). TLR4 enhances TGF-beta signaling and hepatic fibrosis. Nat. Med. 13: 1324-1332. http://dx.doi.org/10.1038/nm1663

Seki E, Park E and Fujimoto J (2011). Toll-like receptor signaling in liver regeneration, fibrosis and carcinogenesis. Hepatol. Res. 41: 597-610. http://dx.doi.org/10.1111/j.1872-034X.2011.00822.x

Sheikhbahaei S, Abdollahi A, Hafezi-Nejad N and Zare E (2014). Patterns of antimicrobial resistance in the causative organisms of spontaneous bacterial peritonitis: a single centre, six-year experience of 1981 samples. Int. J. Hepatol. 2014: 917856. http://dx.doi.org/10.1155/2014/917856

Shimoda S, Harada K, Niiro H, Shirabe K, et al. (2011). Interaction between Toll-like receptors and natural killer cells in the destruction of bile ducts in primary biliary cirrhosis. Hepatology 53: 1270-1281. http://dx.doi.org/10.1002/hep.24194

Shinkai H, Muneta Y, Suzuki K, Eguchi-Ogawa T, et al. (2006). Porcine Toll-like receptor 1, 6, and 10 genes: complete sequencing of genomic region and expression analysis. Mol. Immunol. 43: 1474-1480. http://dx.doi.org/10.1016/j. molimm.2005.09.006

Singh R, Bullard J, Kalra M, Assefa S, et al. (2011). Status of bacterial colonization, Toll-like receptor expression and 
nuclear factor-kappa B activation in normal and diseased human livers. Clin. Immunol. 138: 41-49. http://dx.doi. org/10.1016/j.clim.2010.09.006

Sohn JJ, Schetter AJ, Yfantis HG, Ridnour LA, et al. (2012). Macrophages, nitric oxide and microRNAs are associated with DNA damage response pathway and senescence in inflammatory bowel disease. PLoS One 7: e44156. http:// dx.doi.org/10.1371/journal.pone.0044156

Wang B, Trippler M, Pei R, Lu M, et al. (2009). Toll-like receptor activated human and murine hepatic stellate cells are potent regulators of hepatitis C virus replication. J. Hepatol. 51: 1037-1045. http://dx.doi.org/10.1016/j. jhep.2009.06.020

Wang J and Lu Q (2008). Expression of T subsets and mIL-2R in peripheral blood of newborns with hypoxic ischemic encephalopathy. World J. Pediatr. 4: 140-144. http://dx.doi.org/10.1007/s12519-008-0028-4

Wang J, Zhao JH, Wang PP and Xiang GJ (2008). Expression of CXC chemokine IP-10 in patients with chronic hepatitis B. HBPD INT 7: 45-50.

Wang J, Wang PP, Xiang GJ and Hu XB (2010). Relationship between the expression of IP-10 and IP-10 mRNA in peripheral blood and HBV DNA level in patients with cirrhosis. HBPD INT 9: 280-286.

Wu J, Meng Z, Jiang M, Pei R, et al. (2009). Hepatitis B virus suppresses toll-like receptor-mediated innate immune responses in murine parenchymal and nonparenchymal liver cells. Hepatology 49: 1132-1140. http://dx.doi. org/10.1002/hep. 22751

Wu J, Meng Z, Jiang M, Zhang E, et al. (2010). Toll-like receptor-induced innate immune responses in non-parenchymal liver cells are cell type-specific. Immunology 129: 363-374. http://dx.doi.org/10.1111/j.1365-2567.2009.03179.x

Ye D, Li FY, Lam KS, Li H, et al. (2012). Toll-like receptor-4 mediates obesity-induced non-alcoholic steatohepatitis through activation of X-box binding protein-1 in mice. Gut 61: 1058-1067. http://dx.doi.org/10.1136/gutjnl-2011-300269

Zhang Y, Lin A, Zhang C, Tian Z, et al. (2014). Phosphorothioate-modified CpG oligodeoxynucleotide (CpG ODN) induces apoptosis of human hepatocellular carcinoma cells independent of TLR9. Cancer Immunol. Immunother. 63: 357-367. http://dx.doi.org/10.1007/s00262-014-1518-y

Zhao J, Zhao S, Zhou G, Liang L, et al. (2011). Altered biliary epithelial cell and monocyte responses to lipopolysaccharide as a TLR ligand in patients with primary biliary cirrhosis. Scand. J. Gastroenterol. 46: 485-494. http://dx.doi.org/1 $\underline{0.3109 / 00365521.2010 .539624}$ 